\title{
TCR-mimic bispecific antibodies targeting LMP2A show potent activity against EBV malignancies
}

Mahiuddin Ahmed, ${ }^{1}$ Andres Lopez-Albaitero, ${ }^{1}$ Dmitry Pankov, ${ }^{1}$ Brian H. Santich, ${ }^{1}$ Hong Liu, ${ }^{2}$ Su Yan, ${ }^{2}$ Jingyi Xiang, ${ }^{2}$ Pei Wang, ${ }^{2}$ Aisha N. Hasan, ${ }^{1}$ Annamalai Selvakumar, ${ }^{1}$ Richard J. O'Reilly, ${ }^{1}$ Cheng Liu, ${ }^{2}$ and Nai-Kong V. Cheung ${ }^{1}$

'Department of Pediatrics, Memorial Sloan Kettering Cancer Center, New York, New York, USA. 'Eureka Therapeutics, Emeryville, California, USA.

EBV infection is associated with a number of malignancies of clinical unmet need, including Hodgkin lymphoma, nasopharyngeal carcinoma, gastric cancer, and posttransplant lymphoproliferative disease (PTLD), all of which express the EBV protein latent membrane protein 2A (LMP2A), an antigen that is difficult to target by conventional antibody approaches. To overcome this, we utilized phage display technology and a structure-guided selection strategy to generate human T cell receptor-like (TCR-like) monoclonal antibodies with exquisite specificity for the LMP2A-derived nonamer peptide, $C_{426}$ LCGLLTMV $_{434}$ (CLC), as presented on HLA-A*02:01. Our lead construct, clone 38 , closely mimics the native binding mode of a TCR, recognizing residues at position P3-P8 of the CLG peptide. To enhance antitumor potency, we constructed dimeric T cell engaging bispecific antibodies (DiBsAb) of clone 38 and an affinity-matured version clone 38-2. Both DiBsAb showed potent antitumor properties in vitro and in immunodeficient mice implanted with EBV transformed B lymphoblastoid cell lines and human T cell effectors. Clone 38 DiBsAb showed a stronger safety profile compared with its affinity-matured variant, with no activity against EBV ${ }^{-}$tumor cell lines and a panel of normal tissues, and was less cross-reactive against $H L A-A^{*}$ 02:01 cells pulsed with a panel of CLC-like peptides predicted from a proteomic analysis. Clone 38 was also shown to recognize the CLG peptide on other HLA-A*02 suballeles, including HLA-A*02:02, HLA-A*02:04, and HLA-A*02:06, allowing for its potential use in additional populations. Clone $38 \mathrm{DiBsAb}$ is a lead candidate to treat EBV malignancies with one of the strongest safety profiles documented for TCR-like mAbs.

Authorship note: MA and ALA contributed equally to this work.

Conflict of interest: NKC has ownership in, income from, or research funds from Y-mAbs Therapeutics, Eureka Therapeutics, and AbPro. MA has ownership in and research funds from Y-mAbs Therapeutics. RJO and ANH receive royalties from Atara Biotherapeutics. CL has ownership in Eureka Therapeutics. MA, ALA, CL, HL, SY, JX, PW, and NKC were named as inventors in a patent application for TCR-like antibodies against EBV LMP2A/HLA-A2 filed by Memorial Sloan Kettering Cancer Center, filed under Patent Cooperation Treaty (PCT) WO 2016/201124.

Submitted: September 29, 2017 Accepted: January 10, 2018 Published: February 22, 2018

\section{Reference information:}

JCI Insight. 2018;3(4):e97805 https://doi.org/10.1172/jci. insight. 97805.

\section{Introduction}

Over $90 \%$ of the world's adult population has been infected by EBV (1), and in the absence of immune compromise, initial exposure results in a self-limited illness controlled by a cellular immune response (2). Although most infected individuals can produce potent antigen specific T cells against EBV gene products, the virus can persist in epithelial cells or B cells, and any changes in the immune status of the host can lead to viral reactivation and a number of EBV-associated malignancies (3). It has been estimated that $1.8 \%$ of all cancer deaths worldwide are related to EBV infection (4).

EBV malignancies can be categorized based on one of the 3 infection latency patterns of the host cell: latency I, II, and III (5). A total of 10 latency proteins have been described: 6 Epstein-Barr nuclear antigens (EBNA1, $-2,-3 \mathrm{~A},-3 \mathrm{~B},-3 \mathrm{C}$, and -LP), 3 latent membrane proteins (LMP1, -2A, and -2B), and BARF1 (6). Initial EBV infection activates B cells and induces latency III when EBNA1, EBNA2, EBNA3, LMP1, LMP2, and BARF1 are expressed. Latency III is seen in the setting of solid organ and hematopoietic cell transplantation where the decreased number or absence of T cells may cause unrestricted proliferation of B cells harboring EBV (7). This can result in posttransplant lymphoproliferative disease (PTLD), the most common posttransplant malignancy. In latency II, when EBNA1, LMP1, and LMP2 are expressed, there is a more limited expression of the viral products and conversion of the infected B cell into a memory B cell. Latency II is associated with Hodgkin lymphoma, diffuse large B cell lymphoma (DLBL), NK/T cell lymphoma, and nasopharyngeal carcinoma (NPC). 
Further restriction in the expression of these antigens leads to latency I when only EBNA1 is expressed. Latency I has been associated with Burkitt lymphoma and EBV-associated gastric cancer. Gastric cancer has, however, been more recently categorized as latency I/II and has been shown to express LMP2A $(8,9)$. B cells can have latency programs I, II, or III. However, epithelial cells only present latency I and II. Based on this expression profile, the EBV gene products EBNA1 and LMP2A are considered to be the most important therapeutic targets for EBV-associated malignancies, where EBNA-1 is expressed in all and LMP2A is expressed in most (with the exception of Burkitt's lymphoma) EBV malignancies.

There are limited treatment options for EBV malignancies beyond standard chemotherapy and radiation. One promising modality is adoptive cell therapy using EBV-specific T cells, which has shown efficacy for PTLD (success in 70\% of cases) (6). This type of therapy has utilized polyclonal T cells, which are specific for latency III gene products, particularly the immunodominant EBNA3. Due to the limited expression of antigens in latency I and II, it is unclear whether this approach would be effective for EBV malignancies outside of PTLD.

A more ideal therapeutic agent would be an antibody that can specifically recognize one of the more broadly expressed antigens: EBNA1 or LMP2A. Because of the nuclear localization of EBNA1 and the transmembrane localization of LMP2A (with short extracellular loops), conventional mAbs cannot be easily developed for these targets. An alternative strategy is to develop mAbs that can recognize immunogenic peptides derived from these EBV gene products that are processed and presented on the surface of host cells on HLA class I molecules. This class of antibodies is referred as TCR-like, since they resemble the binding of the TCR to the peptide/HLA complex (pHLA). This approach has been used successfully to target several tumor associated antigens (reviewed in ref. 10). However, the lack of precise epitope mapping for most TCR-like mAbs, poor understanding of how closely the antibodies mimic the endogenous binding mode of TCRs, and little data assessing potential cross-reactivity limit the therapeutic potential of these agents.

We have focused our investigation on generating human mAbs against the pHLA complex corresponding to LMP2A - which is an antigen found on the majority of EBV-related malignancies and ranked no. 3 in the NCI's prioritization of cancer antigens (11) - and the HLA allele HLA-A*02:01. The HLA-A*02 allele is one of the most prevalent in the world (30\% in North America, 29\% in Europe, 29\% in Northeast Asia, and 25\% in Southeast Asia), with HLA-A*02:01 being the most predominant subtype (65\% in North America, $94 \%$ in Europe, 52\% in Northeast Asia, and 28\% in Southeast Asia; ref. 12). We specifically targeted the LMP2A nonamer peptide $C_{426}$ LGGLLTMV $_{434}(C L G)$ as presented on HLA-A*02:01. CLG is an ideal target since it is the most immunogenic HLA-A*02:01 presented peptide (13) within the LMP2A protein and has been successfully used for selection in adoptive T cell therapy (14). Additionally, the LMP2A CLG/HLA-A*02:01 pHLA complex has previously been targeted using a murine TCR-like mAb $(15,16)$; however, as an IgG, this format provided no survival benefit in mouse models of PTLD, and its specificity was not thoroughly assessed beyond showing a lack of reactivity against EBV ${ }^{-} \mathrm{PBMC}$.

Using structure-based analyses and phage display selection strategies, we generated a human TCRlike $\mathrm{mAb}$ that mimics the native binding mode of a TCR, showing exquisite specificity to LMP2A CLG/ HLA-A*02:01 pHLA complex, with no cross-reactivity to a panel of normal tissues and $\mathrm{EBV}^{-}$tumor cell lines. To enhance antitumor potency without sacrificing specificity, we generated a $\mathrm{T}$ cell-engaging bispecific antibody (BsAb) format of our lead construct, resulting in potent antitumor efficacy in vitro and in mouse xenograft studies. We additionally tested the ability of our lead construct to recognize the LMP2A CLG peptide in the context of HLA-A*02 suballeles, for potential use in additional patient populations.

\section{Results}

Structural analysis of TCR binding to peptide/HLA class I complexes. To create TCR-like mAbs that more closely mimicked TCRs, we first investigated how TCRs bind pHLA from known cocrystal structures. We identified 14 unique TCR/nonamer-pHLA class I cocrystal structures from the protein data bank (http://www. rcsb.org/) (Supplemental Table 1; supplemental material available online with this article; https://doi. org/10.1172/jci.insight.97805DS1) (17-23). We only considered native human TCR/pHLA interactions and excluded nonhuman TCRs, unnatural peptide variants, and artificially affinity-enhanced TCRs. We also identified 5 unique TCR-decamer-pHLA class I cocrystal structures from the protein data bank (Supplemental Table 2). Because of the smaller size of the decamer-peptide data set, we included 2 structures with heteroclitic peptides. All of these structures show that TCRs share the previously described (24) common diagonally binding geometry to the peptide in the HLA cleft (Figure 1A). We then undertook a more 
detailed molecular analysis of this interaction by calculating the per-residue interaction energies of the peptide with the bound TCR using Chemistry at Harvard Molecular Mechanics (CHARMM) force field simulations. To understand the short-range molecular contacts at the TCR/pHLA interface, the van der Waals interaction energies were analyzed for each residue of the nonamer and decamer peptides. The van der Waals interaction energies for the nonamer structures show a bell-shaped distribution involving residues at positions $\mathrm{P} 3-\mathrm{P} 8$, with the highest interaction occurring with residue position P5 (Figure 1B). As expected, the least interaction with the TCR occurs with the peptide anchor residues P2 and P9. A similar bell-shaped distribution of interaction energies was observed for the decamer-pHLA structures (Supplemental Figure 1). From this, we concluded that, in order to most closely resemble a native TCR/pHLA interaction, an ideal TCR-like $\mathrm{mAb}$ must interact with the central peptide residues.

Development of human TCR-like mAbs against CLG/HLA- $A^{*}$ 02:01. To generate human anti-pHLA antibodies, we panned an antibody phage display library against the CLG/HLA-A*02:01 complex (see Methods). Phage clones were selected based on affinity and specificity to the CLG/HLA-A*02:01 complex as compared with a panel of 19 irrelevant peptide/HLA-A*02:01 complexes. Four top clones were chosen (clones 26, 38, 40, and 61), with each binding to CLG/HLA-A*02:01 but none of the 19 irrelevant peptides.

To identify the precise binding epitopes of each clone, we generated Ala-substituted variants of the CLG peptide and measured the variation in phage binding by FACS. Initially, the HLA loading efficiency of each Ala-substituted peptide was validated using BB7.2 mAb to stain pulsed T2 cells (Supplemental Figure 2). It was observed that positions $\mathrm{P} 1$ and $\mathrm{P} 2$ did not tolerate Ala-substitution for HLA-A*02:01 loading, likely due to the importance of Cys at P1 and Leu at P2 for anchoring the CLG peptide to the HLA protein. Ala-substitution at the other nonanchor residues (P3-P8) was well tolerated, and the corresponding peptides variants were used to map the epitopes of the top 4 clones (Figure 2A). Clone 38 had the widest epitope coverage, with a bell-shaped distribution spanning positions P3-P8, similar to the native TCRs depicted in Figure 1B. Clones 40 and 61 had similar central spanning epitopes (P4-P8), and clone 26 had an epitope closer to the C-terminus of the peptide (P6-P8).

Next, we sought to evaluate the binding kinetics of each clone in a human IgG1 format. The highest affinity $\left(2 \mathrm{nM} K_{\mathrm{D}}\right)$ was found in $26 \mathrm{IgG} 1$, followed by $61 \mathrm{IgG} 1(26 \mathrm{nM})$, while both $38 \mathrm{IgG} 1$ and $40 \mathrm{IgG} 1$ were lowest (52 nM). Interestingly, $38 \mathrm{IgG} 1$ had the slowest dissociation rate (with a dissociation rate $\left[\mathrm{k}_{\text {off }}\right]$ ) more than 10-fold slower than 26 IgG1, despite having a weaker overall $K_{\mathrm{D}}$ (Figure 2B and Supplemental Table 3).

As IgGs, the 4 clones were then tested for their ability to bind peptide pulsed $\mathrm{T} 2$ cells and mediate antibody-dependent cytotoxicity (ADCC) (Supplemental Figure 3). All 4 clones bound to the T2 cells and displayed ADCC activity, with 38 IgG1 showing the least potency.

To validate the binding activity of each clone against an endogenous HLA-A*02:01+EBV ${ }^{+}$target, RPMI-6666 (EBV ${ }^{+}$BLCL derived from a Hodgkin's lymphoma patient) was incubated with phage from each phage clone (Figure 3). Surprisingly, clone 38 showed robust binding to endogenously expressed CLG/HLA-A*02:01 complexes, whereas clones 26 and 40 showed only modest binding, and clone 61 showed absolutely no binding. We hypothesize that this difference in recognition for RPMI-6666 compared with pulsed T2 cells was a result of heterogeneity in the endogenous processing and presentation of the LMP2A sequence in EBV transformed cells. Based on the robust cell-binding activity against an endogenously expressed target and its wide epitope coverage, clone 38 was chosen as the lead candidate.

We then measured the ADCC activity of 38 IgG1 against several HLA-A*02:01+EBV ${ }^{+}$target cells but saw low potency (data not shown), likely due to insufficient expression and presentation of the CLG/HLA-A*02:01 complexes.

Engineering monomeric bispecific $T$ cell-engaging tandem single-chain variable fragment (scFv) antibodies. To increase the antitumor potency of clone 38, we first reengineered it as a tandem-scFv BsAb, similar to the FDA-approved blinatumomab (25), with the $38 \mathrm{scFv}$ at the N-terminal end and an anti-human $\mathrm{CD} 3 \varepsilon \mathrm{scFv}$ at the C-terminal end. We tested the $38 \mathrm{BsAb}$ for activity against several HLA-A*02 suballeles using artificial antigen presenting cells (aAPCs) that were pulsed with CLG peptide (Supplemental Figure 4). Each aAPC line stably expressed 1 of 6 different HLA-A*02 suballeles: *02:01, ${ }^{*} 02: 02,{ }^{*} 02: 03,{ }^{*} 02: 04,{ }^{*} 02: 05$, or ${ }^{*} 02: 06$. Potent activity against HLA-A*02:01, HLA-A*02:02, HLA-A*02:04, and HLA-A*02:06 was shown by 38 BsAb, with moderate to low activity observed against HLA-A*02:03 and HLA-A*02:05. This demonstrated that recruiting $\mathrm{T}$ cells can greatly improve the activity of this antibody and that clone 38 could be utilized on a population of patients that express these suballeles in addition to HLA-A*02:01. 

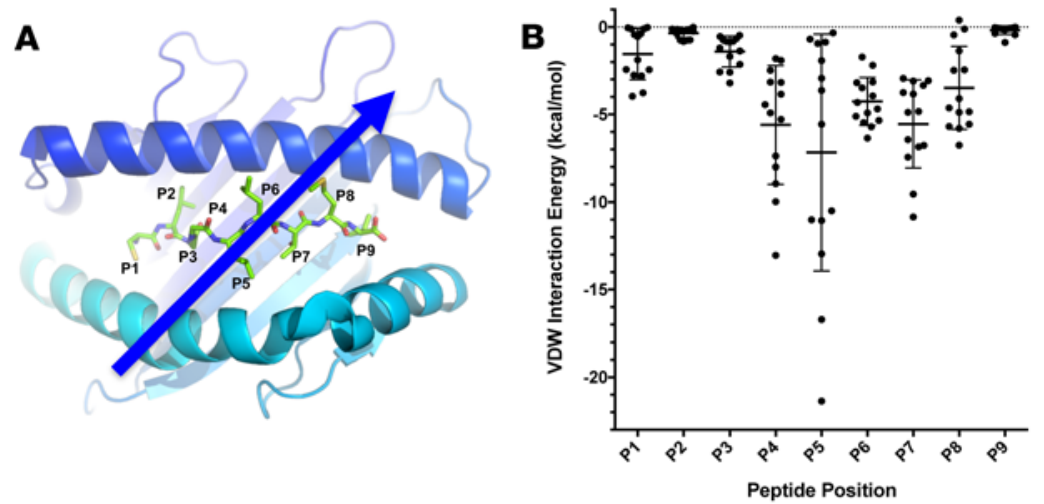

Figure 1. Analysis of TCR/nonamer pHLA complex interactions shows common binding modes. (A) Crystal structure of CLG/HLA-A*02:01 complex with arrow indicating typical diagonal binding by a TCR. Peptide positions are labeled P1-P9. Figure was rendered using the 1.9 A resolution structure from pdb 3REW (http://www.rcsb.org/structure/3REW). (B) Plot of calculated VDW contact energies of known unique TCR/nonamer pHLA class I cocrystal structures showing a bell-shaped distribution of contacts along peptide interface. The peptide position (P1, P2, etc.) is indicated on the $x$ axis of the graph. Individual interaction energies from 14 different structures are plotted, with bars indicating mean $\pm \mathrm{SD}$.

Affinity maturation of clone 38. We had previously seen substantial improvement in potency from increasing affinity to a pHLA target (26), and since clone 38 had modest affinity, we reasoned that it was a good candidate for maturation. Initially, a phage library was generated from clone 38 by random mutagenesis and was repanned against the CLG/HLA-A*02:01 complex. From a total of 581 clones, a final 8 unique clones demonstrated improvement over the parental antibody (Supplemental Table 4). Among these final 8 clones, clone 38-2 had the largest improvement in affinity (1.7-fold) without losing specificity to the CLG/HLA-A*02:01 complex relative to a panel of irrelevant pHLA complexes. Clone 38-2 contains a single point mutation $(\mathrm{P} 55 \mathrm{H})$ in the CDR2 of the variable light $\left(\mathrm{V}_{\mathrm{L}}\right)$ domain.

Generation of optimized dimeric BsAb format. Despite the potency observed by the $38 \mathrm{BsAb}$, the format had poor stability compared with the dimeric BsAb format (DiBsAb) that we had recently developed (27). This dimeric format improves upon the conventional monomeric BsAb format by allowing for bivalent tumor-antigen binding (log-fold increase in affinity) and improved pharmacokinetics. Clones 38 and 38-2 were reengineered into DiBsAbs by mutating a free cysteine residue in the anti-human CD3e scFv (to reduce aggregation) and adding a human homodimerization domain to the C-terminus (Figure 4A). DiBsAbs were found to be exceptionally pure (>95\% pure) and stable under accelerated stress conditions (Supplemental Figure 5).

The in vitro potency of $38 \mathrm{DiBsAb}$ and 38-2 DiBsAb were compared using CLG-pulsed T2 cells (Figure $4 \mathrm{~B})$, as well as $4 \mathrm{HLA}-\mathrm{A}^{*} 02: 01^{+} \mathrm{EBV}^{+}$tumor cell lines: RPMI-6666, DT BLCL, F BLCL, and HONE-1-A2 (NPC cell line transfected with HLA-A*02:01) (Figure 5 and Supplemental Table 5). For the CLG-pulsed T2 cells, 38-2 DiBsAb was more potent and had a 10-fold lower half maximal effective concentration $\left(\mathrm{EC}_{50}\right)$ compared with the parent $38 \mathrm{DiBsAb}\left(0.3 \mathrm{ng} / \mathrm{ml}\right.$ and $3 \mathrm{ng} / \mathrm{ml} \mathrm{EC}{ }_{50}$, respectively). A similar pattern was observed with the 4 tumor cell lines screened. Clone 38-2 DiBsAb had $\mathrm{EC}_{50}$ values of 2-70 $\mathrm{ng} / \mathrm{ml}$ and max killing of $37 \%-55 \%$, while $38 \mathrm{DiBsAb}$ had $\mathrm{EC}_{50}$ values of $10-490 \mathrm{ng} / \mathrm{ml}$ and max killing of $17 \%-40 \%$.

To validate their specificity to the CLG/HLA-A*02:01 complex, 38 and 38-2 DiBsAbs were also screened against several antigen-negative tumor cell lines (Figure 5), including HLA-A*02:01-EBV ${ }^{+}$tumor cell line KS BLCL and HLA-A ${ }^{*} 02^{+} \mathrm{EBV}^{-}$tumor cell lines COLO205 (colorectal cancer), MCF-7 (breast cancer), and HepG2 (hepatocellular cancer). No substantial cytotoxicity was observed compared with the control DiBsAb.

Mouse xenograft studies of DiBsAbs. After showing strong in vitro potency, 38 and 38-2 DiBsAbs were tested in mouse xenograft models of PTLD to measure their in vivo efficacy. In the first study (Figure 6), F BLCLLuc (F BLCL transduced with a luciferase reporter gene) were injected i.v. into immunodeficient BALBRag2---IL-2R- $\gamma \mathrm{c}-\mathrm{KO}(\mathrm{DKO})$ mice and then treated with i.v. injections of adult PBMC $(1 \times /$ week for 2 weeks $)$ and either 38, 38-2, or control DiBsAb (14 injections over 5 weeks). Tumor growth was monitored by bioluminescence (Figure 6B). Luciferase activity saturated at day 28, and AUC was quantified (Supplemental Table 6). 38 and 38-2 DiBsAbs were highly effective and reduced tumor growth (8.8-fold and 38-fold reduction compared with a control DiBsAb, respectively). Median survival for the control antibody group was 39 days, compared with 68 days for $38 \mathrm{DiBsAb}$ treatment $(P=0.04)$ and 70 days for 38-2 DiBsAb treatment $(P=0.03)$.

In order to remove the possibility of endogenous EBV-specific T cells from contributing to the tumor response, we did an additional animal study using human cord blood PBMC (cPBMC) (Figure 7). These cells have not been exposed to exogenous antigens and, thus, should lack any endogenous specificity to EBV-derived pHLA complexes. Similar to the previous experiment, immunodeficient DKO mice were injected i.v. with F BLCL-luc and treated with i.v. injections of cPBMC (1×/week for 2 weeks) and either 38, 38-2, or control DiBsAb (14 injections over 5 weeks). Luciferase activity saturated at day 28 , and AUC was again quanti- 


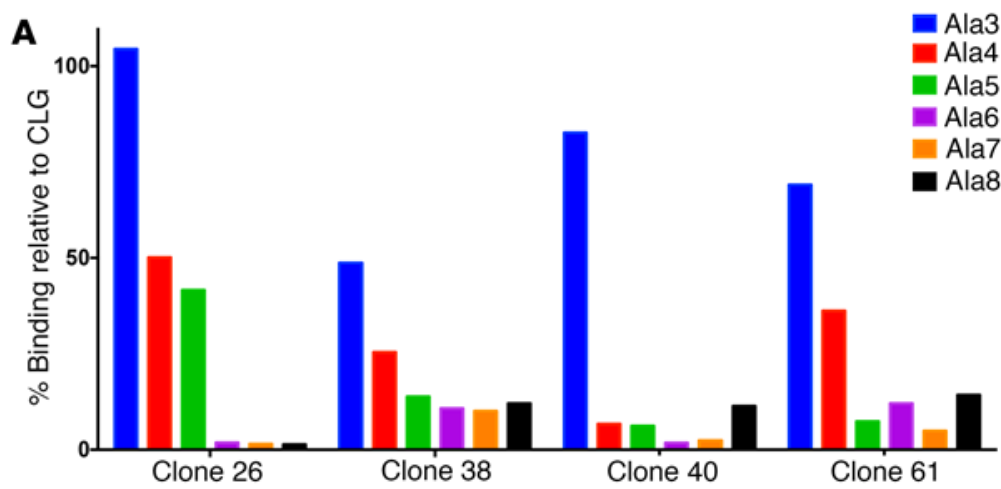

B
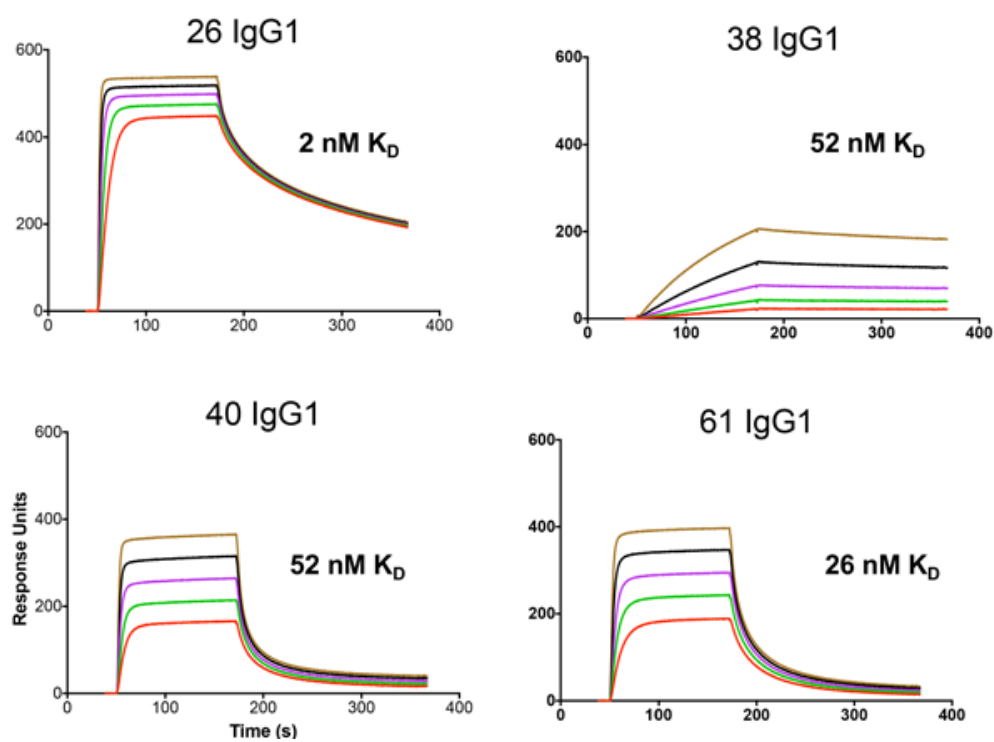

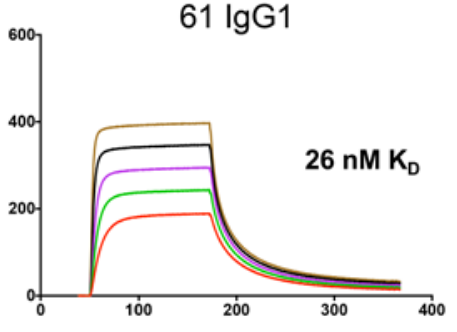

Figure 2. Biochemical analysis of top antibody clones show distinct binding epitopes and affinities. (A) Epitope mapping of top 4 clones $(26,38,40,61)$ based on Ala-substituted CLG peptides at positions P3-P8. Clones were tested in a human IgG1 format for their ability to bind to pulsed T2 cells, as measured by flow cytometry. $T 2$ cells were loaded with either WT CLG peptide or Ala-substituted CLC peptides at positions P3-P8. (B) SPR sensorgrams showing the binding kinetics of top 4 clones (26, 38, 40, 61) in a human IgG1 format. Each sensorgram shows the association and dissociation kinetic curves at the following antibody concentrations: 50 (red), 100 (green), 200 (purple), 400 (black), and 800 (brown) nM. Calculated affinity constants $\left(K_{\mathrm{D}}\right)$ are displayed in each graph.

fied (Supplemental Table 6). In this model, 38 and 38-2 DiBsAbs were also highly effective and reduced tumor growth (4.3-fold and 6.8-fold reduction compared with the control DiBsAb, respectively). Using cPBMC also resulted in a higher survival rate, likely due to lower incidence to graft-versus-host disease from the more naive cPBMC compared with adult PBMC, a phenomenon that has previously been reported (28). Median survival for the control antibody group was 40 days, compared with 83 days for $38 \mathrm{DiBsAb}$ treatment $(P=0.003)$. For the 38-2 DiBsAb treatment group, 3 of 5 mice were still alive after 100 days $(P=0.003)$.

Additional safety and potential cross-reactivity assessment. In addition to screening against 19 irrelevant peptide/HLA-A*02:01 complexes during the initial phage panning and doing $\mathrm{T}$ cell-dependent cytotoxicity (TDCC) against 4 target negative cell lines (Figure 5), we did additional sets of experiments to assess potential cross-reactivity: TDCC assays against normal tissues and TDCC assays against T2 cells pulsed with nonamer peptides derived from endogenous human proteins, which closely resemble the CLG peptide biochemically. Initially, we looked at a commercially available panel of normal cells (Supplemental Figure 6). Of this set, only cardiac myocytes and adrenal cortical cells showed HLA-A*02 expression via BB7.2 staining. 38 DiBsAb showed no significant killing compared with the control DiBsAb, whereas 38-2 DiBsAb showed elevated killing of cardiac myocytes, indicating some cross-reactivity of the affinity-matured construct.

Following this, we identified 12 peptides, from an analysis of the human proteome, which had a match of at least $66 \%$ (6 of 9) residues and at least $80 \%$ (4 of 5) of the epitope (P3-P8) of the CLG peptide. In addition, these peptides were screened computationally (IEDB score) for their propensity to bind to HLA-A*02:01 (29). These peptides were then pulsed onto T2 cells and assayed by TDCC (Supplemental Figure 7 and Supplemental Table 8). Surprisingly, 38 DiBsAb showed killing above background for $33 \%$ of the peptides (4 of 12), and 38-2 DiBsAb showed killing against $75 \%$ of the peptides (9 of 12). This again suggested that the affinity-matured $38-2 \mathrm{scFv}$ was more cross-reactive than the parental $38 \mathrm{scFv}$. 
Clone 26

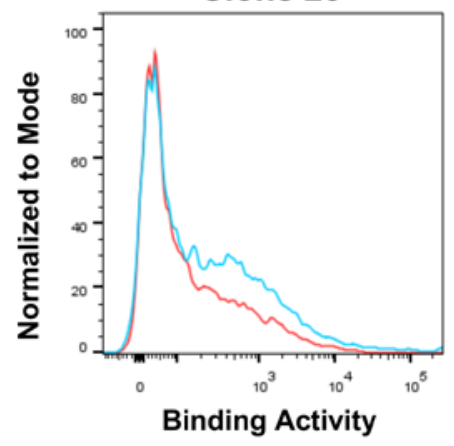

Clone 38

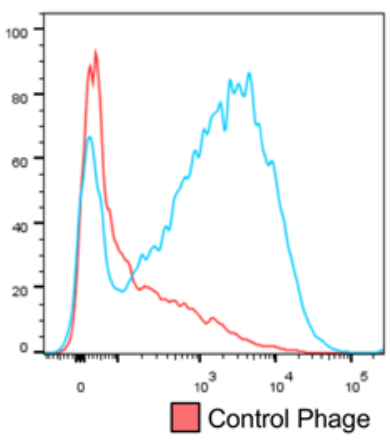

Clone 40

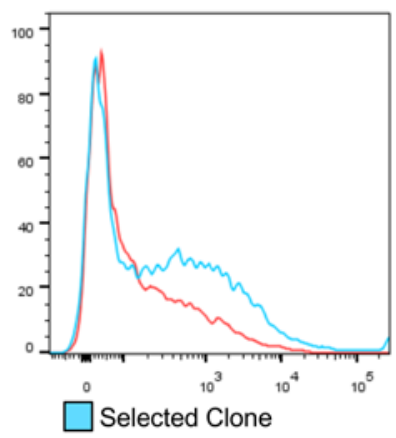

Clone 61

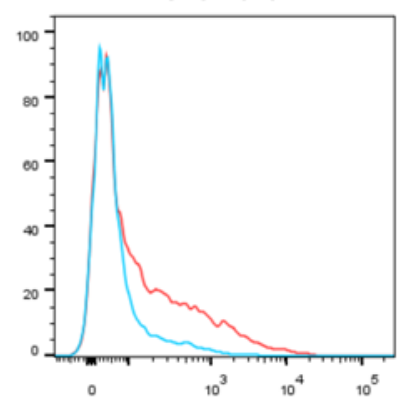

Figure 3. Clone $\mathbf{3 8}$ has highest level of binding to EBV+HLA-A*02:01+ tumor cell target. The binding activity of the top 4 clones was measured by flow cytometry using cell line RPMI-6666. Cells were directly stained with phage from each clone for better signal/noise ratio. Phage were detected with an anti-M13 antibody. Red denotes a control phage, while blue denotes the selected clone.

We next sought to assess if the 4 potential cross-reactive peptides for $38 \mathrm{DiBsAb}$ are actually presented endogenously on HLA-A*02:01+ cells and if $38 \mathrm{DiBsAb}$ binds to them. The 4 cross-reactive peptides come from the gene products of ARMC4, CLDN14, LILRB5, and ORMDL2, respectively, and we identified a number of cell lines that reportedly express them (Supplemental Table 9). ARMC5 and ORMDL2 are reported to be expressed on the HLA-A*02:01+ cell lines MCF7 and U2OS, of which MCF-7 had previously showed no cytotoxicity from 38 or 38-2 $\mathrm{DiBsAb}$ (Figure 5). We additionally tested U2OS and found no killing above background (data not shown). The gene product of CDLN14 is reported to be expressed on liver cells; however, we did not see cytotoxicity against HLA-A*02:01+ HepG2. The last target, LILRB5, is known to be expressed on leukocytes, so we performed TDCC against $2 \mathrm{HLA}-\mathrm{A}^{*} 02^{+}$donor PBMC and found no significant cytotoxicity from 38 or 38-2 $\mathrm{DiBsAb}$ (data not shown). We therefore found no evidence that these 4 potential cross-reactive peptides, which each differ from the core GLLTM epitope by a single amino acid residue, are actually processed and presented on HLA. The data indicates that this small set of potentially cross-reactive GLLTM-like epitopes is not likely presented in the human HLA-peptidome.

\section{Discussion}

Using phage display technology, we have generated human mAbs specific for the antigenic CLG peptide derived from the EBV-associated protein LMP2A in the context of HLA-A*02:01. While our antibodies were originally generated against the peptide in complex with HLA-A*02:01, a predominant allele in North America and Europe, we have shown that these antibodies can also recognize the CLG peptide in certain other HLA-A*02 variants, opening up the possibility for their use in additional populations globally. Our lead TCR-like mAb has been selected not only for its affinity and specificity for the CLG/HLA-A*02:01 complex, but also for its ability to bind key amino acid residues within this sequence. Clone 38 is the first example of a TCR-like mAb that mimics the native binging mode of a TCR, with a bell-shaped distribution of contacts along the peptide interface, centered on peptide position P5, and covering the span of peptide positions P3-P8. When formatted as a DiBsAb, $38 \mathrm{DiBsAb}$ and its affinity-matured variant, 38-2 DiBsAb, had potent antitumor activity against $\mathrm{EBV}^{+} \mathrm{HLA}-\mathrm{A}^{*} 02: 01^{+}$tumor cells. We additionally saw potent antitumor activity in mouse models of PTLD, where immunodeficient mice were xenografted with $\mathrm{EBV}^{+} \mathrm{HLA}-\mathrm{A}^{*} 02: 01^{+} \mathrm{BLCLs}$ and treated with DiBsAb and human adult or cPBMC. While the 38 and 38-2 $\mathrm{DiBs} A \mathrm{~b}$-treated mice had improved survival compared with control groups, the dosing schedule and relatively short serum half-life of the DiBsAb format (estimated $t_{1 / 2}$ of 1 hour based on previous study; ref. 27) may have been suboptimal to achieve higher cure rates. Nevertheless, the 38 and 38-2 DiBsAbs are human antibodies that displayed highly potent killing of $\mathrm{EBV}^{+}$tumors.

Previous attempts to generate TCR-like $\mathrm{mAbs}$ against EBV antigen based on mouse immunization strategies $(15,16)$ resulted murine mAbs against peptides derived from LMP1, LMP2 (CLG), and EBNA-1, in the context of HLA-A*02:01 and related suballeles (12). Of these antibodies, only the EBNA-1 TCR-like mAb was shown to modestly improve survival in immunodeficient mice implanted with $\mathrm{EBV}^{+} \mathrm{BLCLs}$. However, the binding epitope was not investigated and no evidence of potential cross-reactivity was presented, other 
A

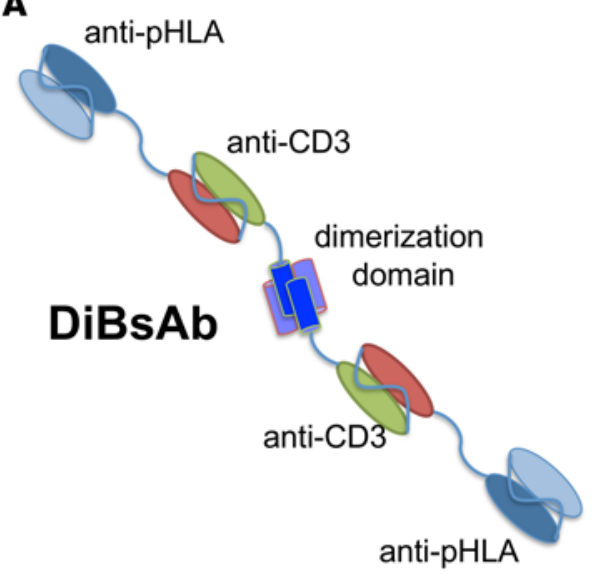

B

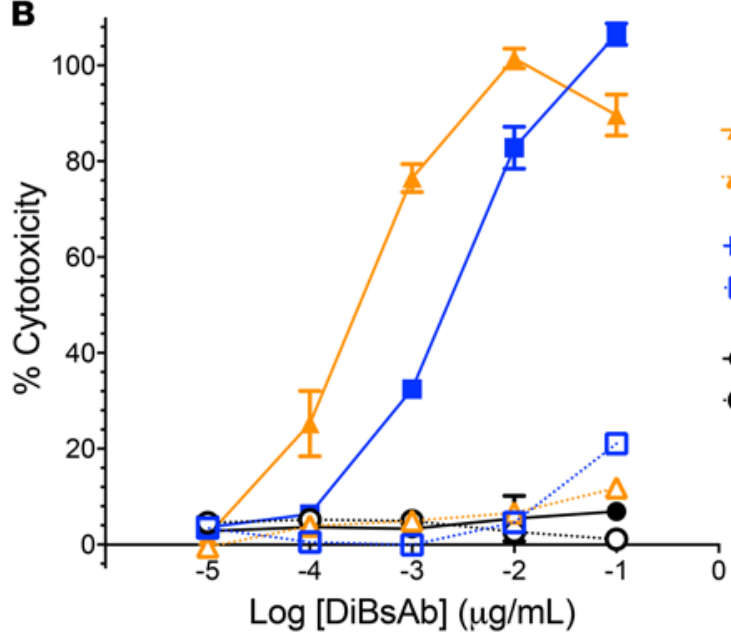

- 38-2 DiBsAb (CLG)

. $38-2 \mathrm{DiBsAb}(\mathrm{YML})$

38 DiBsAb (CLG)

$38 \mathrm{DiBsAb}(\mathrm{YML})$

Control DiBsAb (CLG)

- Control DiBsAb (YML)

Figure 4. Clone 38 and affinity-matured clone 38-2 were engineered into dimeric $\mathrm{T}$ cell-engaging bispecific antibodies with potent activity against CLG-pulsed T2 cells. (A) Schematic of the dimeric T cell engaging bispecific antibody (DiBsAb) format. Two tandemly linked scFvs are linked to a human homodimerization domain, which facilitate self-assembly into a homodimeric complex. (B) TDCC plots for clones 38 (blue squares), $38-2$ (orange triangles), and control (black circles) DiBsAbs. Activated cord blood T cells were incubated for 4 hours with T2 cells (10:1 effector/target ratio [E:T]) pulsed with either CLG (LMP2) or YML (irrelevant) peptides. Data are from 3 technical replicates per experimental condition with mean $\pm \mathrm{SD}$ plotted. $\mathrm{EC}_{50}$ values were determined using a nonlinear fitting algorithm (log [agonist] vs. response-variable slope with 4 parameters). The DiBsAb 38-2 was more potent and had a 10 -fold lower $\mathrm{EC}_{50}$ compared with the parent $38 \mathrm{DiBsAb}\left(0.3 \mathrm{ng} / \mathrm{ml}\right.$ and $3 \mathrm{ng} / \mathrm{ml} \mathrm{EC}_{50}$, respectively).

than the lack of recognition of EBV-HLA-A*02:01+ PBMC. Because TCR and TCR-like therapeutics have a high risk of cross-reacting with peptides similar to their intended target $(30,31)$, we undertook extensive investigations into safety and potential cross-reactivity. A typical assessment of potential cross-reactivity involving an antibody therapeutic would involve staining a panel of normal tissues by IHC. Due to the low antigen density of specific pHLA and the high potency of a DiBsAb format, such an analysis would underestimate the potential for toxicity. A more sensitive assay is to look at antibody-dependent T cell-mediated cytotoxicity. Our lead construct $38 \mathrm{DiBsAb}$ was not found to be cytotoxic against 7 different sets of HLA-A*02+ cells: normal cardiac myocytes, normal adrenal cortical cells, normal PBMCs, a breast cancer tumor cell line (MCF-7), a liver cancer cell line (HepG2), an osteosarcoma cell line (U2OS), and a colon carcinoma cell line (COLO205). Using an in silico- and proteomics-based approach, we also identified and tested 12 potentially cross-reactive peptides from the human proteome that were the most similar to the CLG peptide (6/9 total identical residues and 4/5 identical residues in the core epitope P3-P8). Upon investigation, $38 \mathrm{DiBsAb}$ mediated cytotoxicity against 4 of 12 peptides when pulsed on T2 cells. However, further analysis of these 4 cross-reactive peptides revealed that, although the parental protein of each peptide was expressed in HLA-A*02:01 ${ }^{+}$cell types used as targets, no cytotoxicity against the targets was detected, suggesting that these specific peptides may not be processed and presented by HLA. Not surprisingly, the affinity-matured 38-2 DiBsAb was found to have increased cross-reactivity and was, thus, not considered for further development. By these analyses, $38 \mathrm{DiBsAb}$ shows one of the strongest safety profiles ever demonstrated for a TCR-like mAb, a result of our selection strategy to mimic the native binding mode of a TCR. TCR-like mAbs that do not bind like native TCRs (binding to the $\mathrm{N}$ - or $\mathrm{C}$-terminus of the presented peptide instead of the central residues) have been shown to cross-react to multiple off-target peptides and even bind normal PBMC $(26,30)$.

Our findings support the concept that TCR-like mAbs can be generated against previously "undruggable" targets. A large portion of the human proteome is not expressed on the cell surface or is hidden within membrane structures, making it difficult to target with traditional mAbs. Antigenic peptides derived from these proteins, however, can be adequately presented on the cell-surface HLA complexes where they can be targeted by TCR-like mAbs (32-36). These pHLA complexes contain a short peptide sequence that can vary in length but is usually 9 amino acids for class I alleles $(37,38)$. Within these nonamers, there are usually 2 anchoring residues that are located in position 2 and 9 (P2 and P9) for the HLA-A*02:01 allele (39). Cocrystal structures of several TCR/pHLA complexes have allowed for a better understanding of the interaction of these 3 members of the complex (peptide, HLA, TCR) at the immunological synapse, allowing several gener- 
$\mathrm{A} 2(+) / \mathrm{EBV}(+): \mathrm{F} \mathrm{BLCL}$
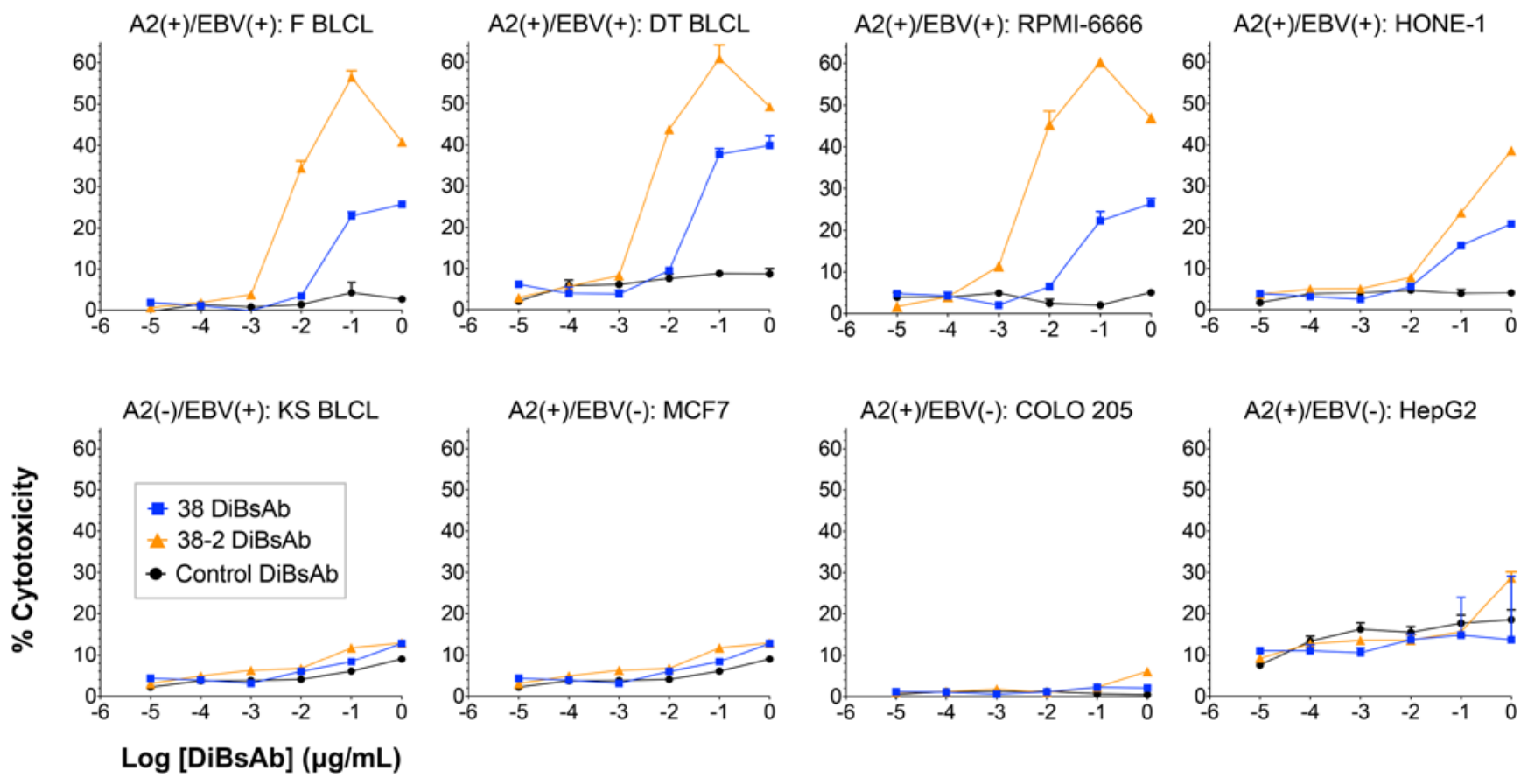

A2(+)/EBV(-): MCF7

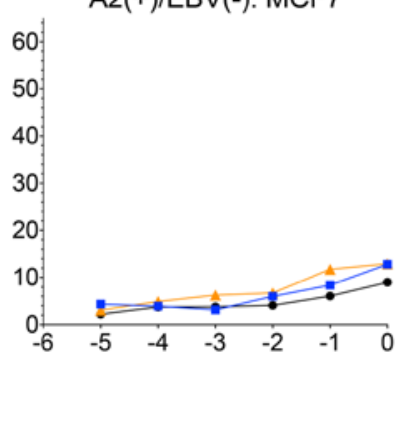

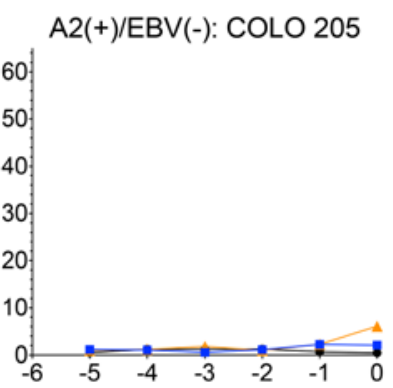

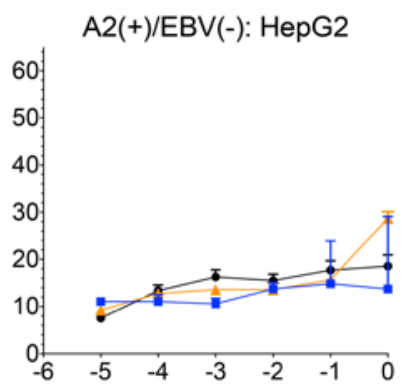

Figure 5. DiBsAbs 38 and 38-2 show potent T cell dependent cytotoxicity of EBV+HLA-A*02:01+ tumor cell targets but not EBV- or $\mathrm{HLA}^{+} \mathrm{A}^{*} 02: 01^{-}$targets. Cytotoxicity and specificity of clone 38 (blue squares) and 38-2 (orange triangles) and control (black circles) DiBsAb was measured against 8 cell lines. Activated cord blood T cells were incubated with each cell line for 4 hours (10:1 E:T). Each curve represents the cytotoxicity of a serial titration of each DiBsAb against $\mathrm{HLA}-\mathrm{A}^{*} \mathrm{O2}: 01^{+} \mathrm{EBV}{ }^{+}$tumor cell lines (top row), and target negative cell lines (bottom row). $\mathrm{HLA}-\mathrm{A}^{*} 02: 01^{+} \mathrm{EBV}^{+}$cell lines: RPMI-6666, DT BLCL, F BLCL, and HONE-1-A2. Target negative cell lines: K BLCL [HLA-A $\left.{ }^{*} 02: 01^{-} E B V+\right], ~ M C F 7, ~ C O L O 205$, and HEPC2 [HLA-A*02:01+EBV-]. Data are from 3 technical replicates per experimental condition with mean $\pm \mathrm{SD}$ plotted. $\mathrm{EC}_{50}$ values were determined using a nonlinear fitting algorithm (log [agonist] vs. response-variable slope with 4 parameters) and are shown in Supplemental Table 5.

alizations. For example, the TCR in $\mathrm{CD} 8^{+} \mathrm{T}$ cells binds to the pHLA complex diagonally at an average angle of 35 degrees with several interaction sites carried by these 3 members of the complex (37). However, $\mathrm{T}$ cell activation itself depends on only a few contact amino acids that are indispensable for mounting a successful immune response. These primary and secondary contact sites are determined by the side chains of 2-5 amino acid residues that are located in the middle portion of the antigenic peptide (40), the so-called functional hotspots $(41,42)$. In the case of nonamers (the most frequent peptide length presented in class I alleles), the most important residue for TCR interaction is located in position 5 of the antigenic peptide (P5), with P6, P7 and $\mathrm{P} 8$ playing secondary roles $(38,40,41)$. In octamer peptides, the most relevant residues are $\mathrm{P} 4, \mathrm{P} 6$, and P7 $(38,40,41)$. Besides the structural evidence, the functional significance of these regions within the antigenic peptide has been consistently proven. For example, EBV-specific memory $\mathrm{T}$ cell activation can occur with as little as 1 amino acid, as long as this residue is one of the primary contact sites (42).

Since Andersen et al., first produced a mAb with TCR specificity (43), there have been over 60 TCRlike mAbs generated against HLA class I complexes. For most of these TCR-like mAbs, it is not clear which specific portions of their target peptide are crucial for recognition $(33,34,36,37,44-57)$. Thus, even though these mAbs recognize a given pHLA, it may not be entirely specific, and any cross-reactivity against similar pHLA can lead to severe or lethal consequences when used in patients $(31,58)$. Furthermore, the mechanisms dictating peptide processing and presentation are not well understood. While we now have more advanced methods for identifying antigenic peptides presented by HLA alleles in an unbiased manner, the generation of TCR-like mAb usually employs peptide pulsing and peptide overexpression, which may influence the way an antigenic peptide is presented and thereby alter the TCR-like antibody's recognition of endogenously processed peptides as they are presented in vivo (59).

In this study, we have generated a lead clone that mimics the native binding mode of a TCR and has exquisite specificity for the endogenously processed LMP2A peptide on $\mathrm{EBV}^{+} \mathrm{HLA}-\mathrm{A}^{*} 02: 01^{+}$targets. While we have demonstrated a high in vitro safety profile for $38 \mathrm{DiBsAb}$, additional in vivo safety testing will need to be per- 
A

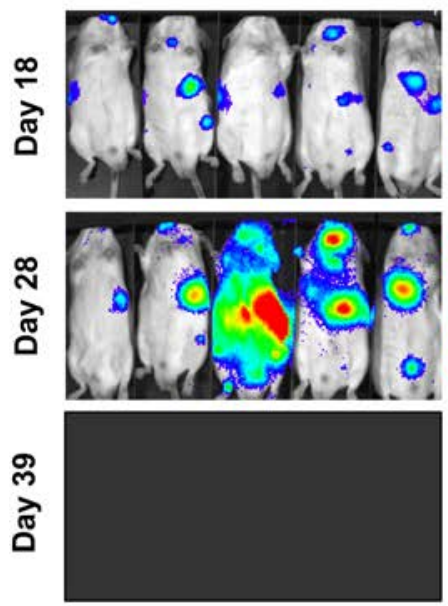

B

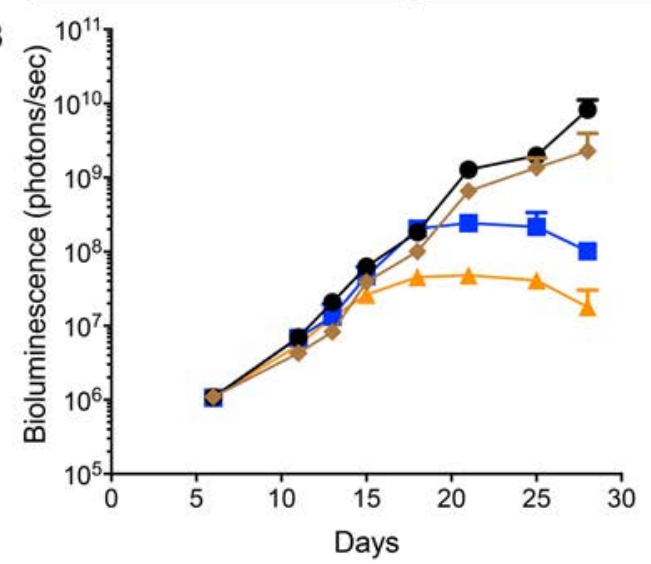

PBMC + Control DiBsAb
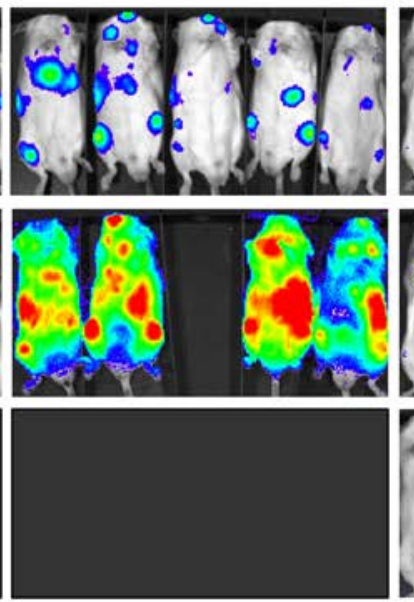

PBMC + 38 DiBsAb
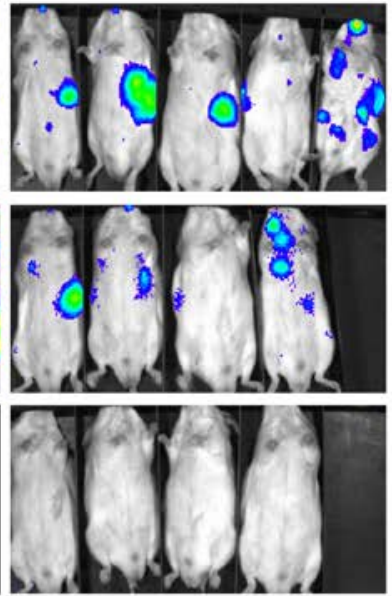

c

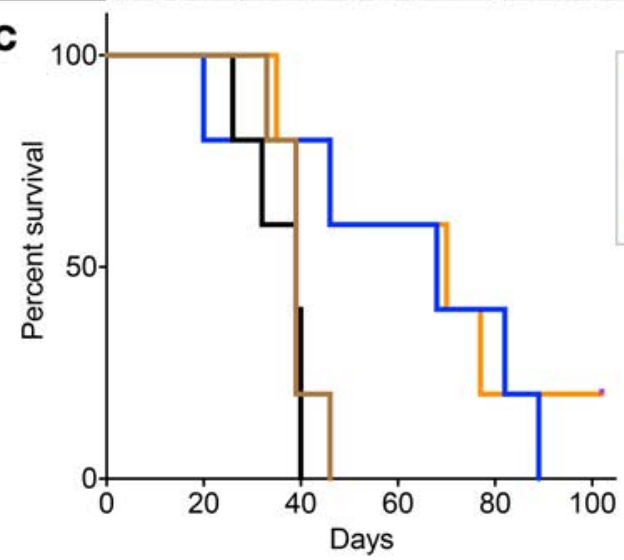

PBMC + 38-2 DiBsAb

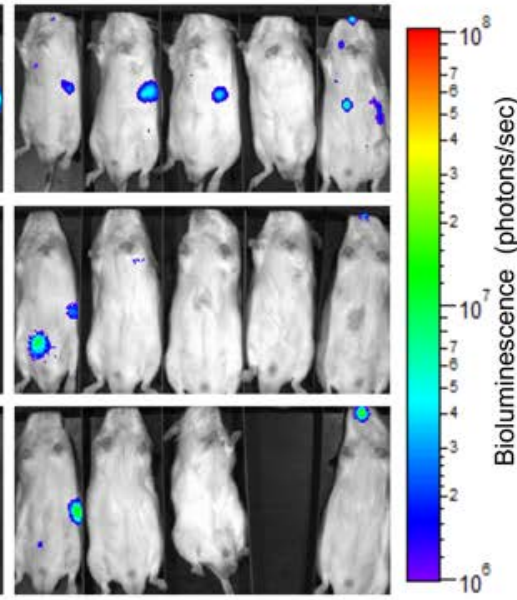

^ $\mathrm{PBMC}$

- PBMC + Control DiBsAb

- PBMC + 38 DiBsAb

- $\mathrm{PBMC}+38-2 \mathrm{DiBsAb}$

Figure 6. DiBsAbs 38 and 38-2 show potent antitumor effect in mouse xenograft study with BLCL and adult PBMC. Immunodeficient DKO mice ( $n=5$ mice per group) were injected i.v. with $1 \times 10^{6} \mathrm{~F}$ BLCL-Luc at day 0 (do) followed by 2 injections i.v. of $10 \times 10^{6}$ human adult PBMC at d7 (50\% T cells) and d14 ( $50 \%$ T cells). Mice were left untreated or treated with $20 \mu$ injections of either control, 38, or 38-2 DiBsAb on days 7, 8, 9, 10, 11, 14, 15, 17, 19, 22, 25, 28, 32, and 39. (A) Bioluminescence images shown for d18, d28, and d39. (B) Quantitation of luciferase activity showing tumor growth and then response to 38 and 38-2 DiBsAb. Data is plotted as mean \pm SEM. AUC analyses and statistical significance are shown in Supplemental Table 6. (C) Kaplan Meyer curves showing survival of each treatment group over time. No-antibody group is shown in brown, control DiBsAb in black, 38 DiBsAb in blue, and 38-2 DiBsAb in orange. Log-rank test was used to determine significance for Kaplan-Meier survival analysis. Median survival for the control antibody group was 39 days, compared with 68 days for 38 DiBsAb treatment $(P=0.04)$ and 70 days for 38-2 DiBsAb treatment $(P=0.03)$.

formed using more relevant animal models. This can include toxicity studies using HLA-A*02:01 transgenic mice (60). While such a system would not mimic the human peptidome that could be processed and presented on the surface of HLA, it would represent a starting point for looking at potential toxicity in humans.

Our lead $38 \mathrm{DiBsAb}$ construct is the most potent antibody therapeutic ever developed to our knowledge to target EBV-related malignancies. EBV-related tumors result in over 140,000 deaths worldwide each year, and our new BsAb therapeutic is a promising candidate for clinical development for a large portion of these patients that are HLA-A* $02^{+}$. We can additionally utilize the same epitope-based selection strategy to develop new therapeutic agents for additional HLA types and for additional antigens. By focusing on specificity and precise epitopes, we chose a lead construct that was not amongst the highest-affinity clones available in our selection pool but resulted in the highest safety profile and ultimately led to the most suitable candidate to advance toward first-in-human trials.

\section{Methods}

Structural analysis. Molecular modeling and interaction energy calculations were done using Discovery Studio 4.1 (Biovia). The crystal structures of TCR/pHLA complexes were energy-minimized using CHARMM force fields simulations, and the atomic interaction energies were then calculated as the sum of van der Waals and electrostatic energies from MM-GBSA simulations. 
A cPBMC
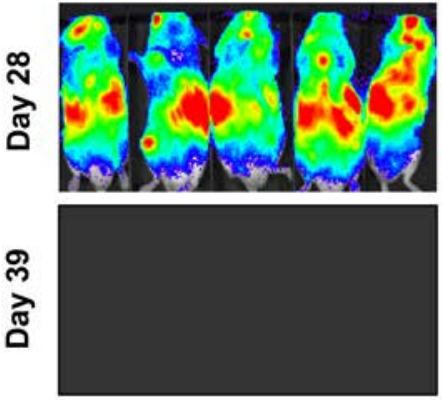

B

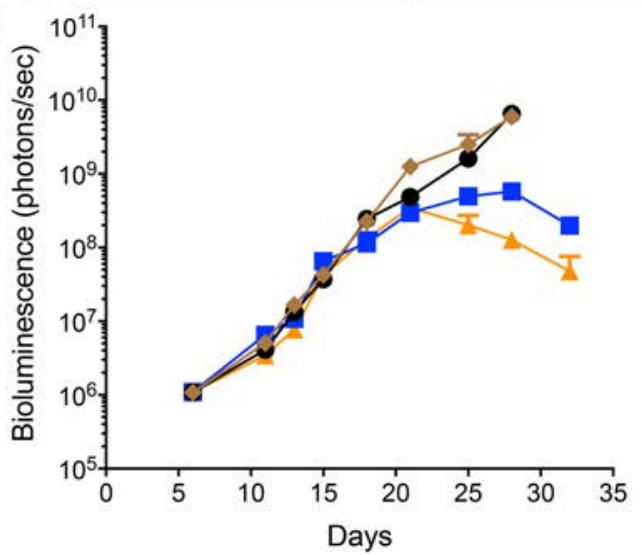
cPBMC + Control DiBsAb

cPBMC + 38 DiBsAb
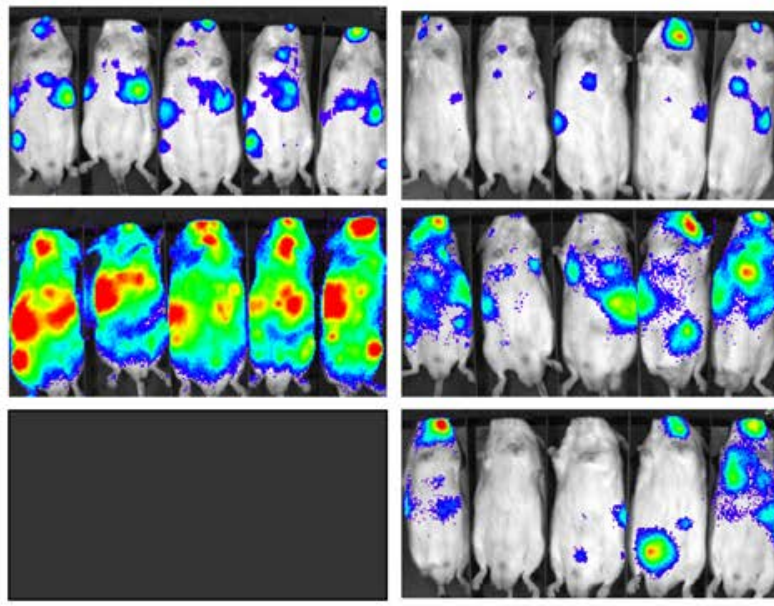

cPBMC + 38-2 DiBsAb

C

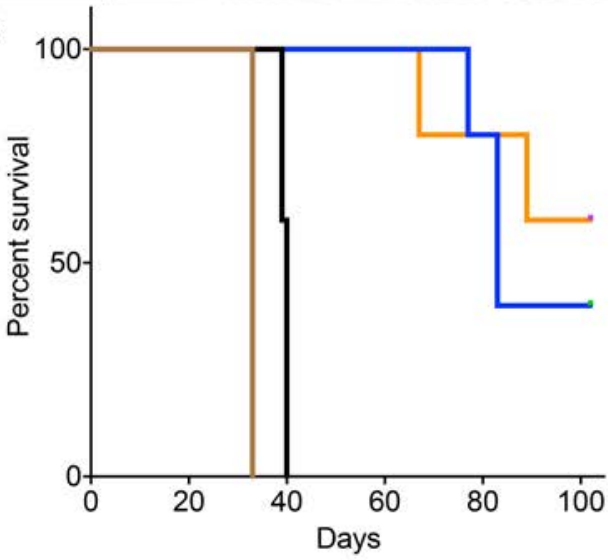

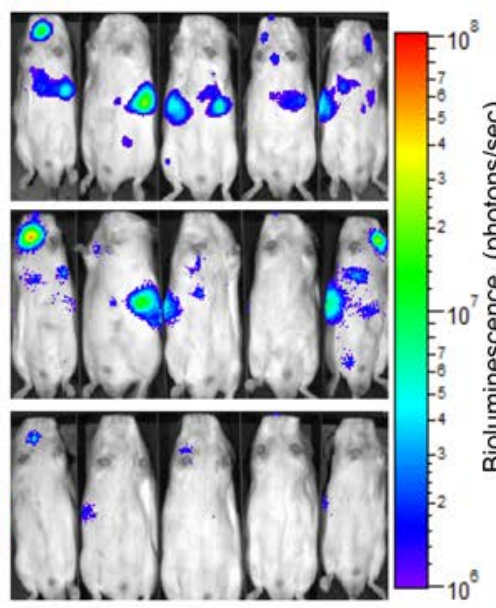

Figure 7. DiBsAbs 38 and 38-2 show potent antitumor effect in mouse xenograft study with BLCL and cord blood PBMC. Immunodeficient DKO mice ( $n=$ 5 mice per group) were injected i.v. with $1 \times 10^{6} \mathrm{~F} \mathrm{BLCL}$-Luc at do followed by 2 i.v. injections of $10 \times 10^{6}$ human cord blood PBMC at d7 (20\% T cells) and d14 ( $50 \%$ T cells). Mice were left untreated or treated with $20 \mu \mathrm{g}$ injections of either control, 38, or 38-2 DiBsAb on days 7, 8, 9, 10, 11, 14, 15, 17, 19, 22, 25, 28, 32, and 39. (A) Bioluminescence images shown for d18, d28, and d39. (B) Quantitation of luciferase activity showing tumor growth and then response to 38 and 38-2 DiBsAb. Data is plotted as mean \pm SEM. AUC analyses and statistical significance are shown in Supplemental Table 7. (C) Kaplan Meyer curves showing survival of each treatment group over time. No-antibody group is shown in brown, control DiBsAb in black, 38 DiBsAb in blue, and 38-2 DiBsAb in orange. Log-rank test was used to determine significance for Kaplan-Meier survival analysis. Median survival for the control antibody group was 40 days, compared with 83 days for 38 DiBsAb treatment $(P=0.003)$. For the 38-2 DiBsAb treatment group, 3 of 5 mice were still alive after 100 days $(P=0.003)$.

Production of biotinylated peptide/HLA-A0201 complex monomer. Biotinylated peptide/HLA-A0201 complex monomers were prepared according to standard protocols (61). In brief, DNA of full-length human $\beta 2 \mathrm{~m}$ was synthesized by Genewiz and cloned into vector pET-27b. The BirA substrate peptide (BSP) was added to the C-terminus of HLA-A0201 extracellular domain (ECD). DNA of HLA-A0201 ECD-BSP was synthesized by Genewiz and cloned into vector pET-27b. The vectors expressing human $\beta 2 \mathrm{~m}$ and HLA-A*02:01 ECD$\mathrm{BSP}$ were transformed into $E$. coli BL21 separately and were isolated as inclusion bodies from bacterial culture. Peptide ligand EBV-LMP2A(CLG)-(full nonamer sequence CLGGLLTMV) was refolded with human $\beta 2 \mathrm{~m}$ and HLA-A*02:01 ECD-BSP to form EBV-LMP2(CLG)/HLA-A*02:01 complex monomer. Folded peptide/ HLA-A*02:01 monomers were concentrated by ultrafiltration and further purified through size-exclusion chromatography. Purified peptide/HLA-A*02:01 monomer was also visualized through SDS-PAGE and biotinylated via BirA-mediated enzymatic reaction and subsequently purified by high-resolution anion-exchange chromatography. Biotinylated peptide/HLA-A*02:01 monomers were stored in PBS at $-80^{\circ} \mathrm{C}$.

HiPrep 26/60 Sephacryl S-300 HR was equilibrated with Hyclone Dulbecco's Phosphate Buffered Saline solution (Thermo Fisher Scientific, catalog SH3002802) for 1.5 column volumes. The unpurified sample was loaded and eluted for 1 column volume. The first peak, consisting of misfolded aggregates, eluted at approximately $102.24 \mathrm{ml}$ after loading. The peak corresponding to the properly folded MHC complex was observed at $201.24 \mathrm{ml}$. Lastly, the peak consisting of free $\beta 2 \mathrm{M}$ was observed at $254.85 \mathrm{ml}$. 
Screening of phage scFv specific for EBV-LMP2(CLG)/HLA-A0201 complex. A human scFv antibody phage display library $\left(10 \times 10^{10}\right.$ clones) constructed by Eureka Therapeutics (E-ALPHA phage library) was used for the selection of human mAbs specific to EBV-LMP2(CLG)/HLA-A*02:01. In order to reduce the conformational change of HLA complex introduced by immobilizing the protein complex onto plastic surfaces, solution panning was used in place of conventional plate panning. In solution panning, biotinylated antigens were first mixed with the human $\mathrm{scFv}$ phage library after extended washing with PBS buffer, and then antigen-scFv antibody phage complexes were pulled down by streptavidin-conjugated Dynabeads M-280 (ThermoFisher) through a magnetic rack. The bound clones were then eluted and used to infect $E$. coli XL1-Blue (Agilent). The phage clones expressed in bacteria were then purified. The panning was performed for 3-4 rounds to enrich $\mathrm{scFv}$ phage clones binding to EBV-LMP2(CLG)/HLA-A*02:01 specifically. Positive clones were determined by standard ELISA method against biotinylated EBV-LMP2(CLG)/HLA-A*02:01 complexes. The positive clones were further tested for their binding to HLA-A2/peptide complexes on live cell surfaces by flow cytometry, using a TAP-deficient, HLA-A0201+ cell line, T2. Briefly, peptide pulsed T2 cells were stained with purified scFv phage clones, and followed by mouse anti-M13 mAb, and R-PE conjugated horse anti-mouse IgG from Vector Labs. Each step of the staining was done on ice with 30-60 minutes of incubation. Cells were washed twice between each step of the staining. A total of 125 positive clones were identified through FACS analysis out of 281 clones screened. Of these 125 clones, 80 unique clones were isolated.

Full-length human IgG1 of the selected phage clones were produced in HEK293 and CHO cell lines, as previously described (62). In brief, antibody variable regions were subcloned into mammalian expression vectors, with matching human lambda or kappa light-chain constant region and human IgG1 constant region sequences. Molecular weight of the purified full-length IgG antibodies was measured under both reducing and nonreducing conditions by electrophoresis.

Cloning of BsAb. The DNA fragments coding for the EBV-LMP2(CLG) scFv antibody and the anti-human $\mathrm{CD} 3 \varepsilon \mathrm{scFv}$ antibody were synthesized by Genewiz and subcloned into Eureka's mammalian expression vector pGSN-Hyg using standard DNA technology. A hex-histamine tag was inserted downstream of the EBV-LMP2(CLG) BsAbs at the C-terminal end for antibody purification and detection. $\mathrm{CHO}$ cells were transfected with the EBV-LMP2(CLG) BsAbs expression vector, and stable expression was achieved by standard drug selection with methionine sulfoximine (MSX), a glutamine synthetase-based (GS-based) method. CHO cell supernatants containing secreted EBV-LMP2(CLG) BsAb molecules were collected. EBV-LMP2(CLG) BsAb was purified using HisTrap HP column (GE Healthcare) by FPLC AKTA system. Briefly, CHO cell culture was clarified and loaded to the column with low imidazole concentration $(20 \mathrm{mM})$, and then an isocratic high imidazole concentration elution buffer $(500 \mathrm{mM})$ was used to elute the bound EBV-LMP2(CLG) BsAb proteins. Molecular weight of the purified EBV-LMP2(CLG) BsAbs were measured under nonreducing conditions by electrophoresis

Affinity determination by surface plasmon resonance. Biotinylated CLG/HLA-A*02:01 complexes were indirectly immobilized onto CM5 sensor chips and analyzed by surface plasmon resonance (SPR) (Biacore, GE Healthcare). Serial dilutions of each clone in a human IgG1 format (50-800nM) were injected at flow rate of $30 \mu \mathrm{l} / \mathrm{min}$ using running buffer (10 mM HEPES, $150 \mathrm{mM} \mathrm{NaCl}, 3 \mathrm{mM}$ EDTA, and 0.05\% Surfactant P-20, pH 7.4) (GE Healthcare). The association and dissociation phase data were fitted simultaneously using a 1:1 model from BIAevaluation 3.2. All the experiments were done at $25^{\circ} \mathrm{C}$.

Cell lines. RPMI-6666, MCF7, COLO205, HepG2, U2OS, and T2 cells were purchased from ATCC. All cells were authenticated by short tandem repeat (STR) profiling (MSKCC Integrated Genomics core facility) and were and periodically tested for mycoplasma (MSKCC Antibody and Bioresource core facility). Normal human cells were purchased from ScienCell. HONE-1 was a gift from G.S.W. Tsao (University of Hong Kong, Pokfulam, Hong Kong, China). HONE-1 is an extensively studied NPC cell line and was authenticated by STR profiling. The HONE-1 line may contain trace amounts of $\mathrm{HeLa}$ originating from the establishment of the line, as noted in the literature (63). For this study, HONE-1 was stably transfected with HLA-A*02:01. EBV infection/transformation of resting B cells produced B-lymphoblastoid cell lines (BLCL) and were generated by the lab of Richard O'Reilly at MSKCC. This process has been accurately replicated in vitro with the use of the B95-8 strain of EBV present in the marmoset B-lymphoblastoid line B95-8 (64). BLCLs present EBV in latent replication and carry multiple copies of the viral genome as an episome and express a number of viral gene products that vary according to latency stage. Some BLCLs were engineered to a express high level of GFP-luciferase fusion protein with retroviral vectors containing a plasmid encoding Luc/GFP. Cells showing high-level GFP expression were selected by flow cytometry analysis and were used for the animal studies. aAPCs expressing various HLA alleles were also generated by the lab of Richard O’Reilly at MSKCC (65). 
Human PBMCs and T cells. Cord blood or adult buffy coats were obtained from the New York Blood Center (New York, New York, USA), and PBMCs were isolated by Ficoll separation. T cells were purified by negative selection (Miltenyi Biotec) and were expanded using CD3/CD28 beads (Invitrogen) following the manufacturer's instructions in 2 rounds of stimulation.

Peptides and peptide pulsing. Peptides were purchased from Genscript and were at least 95\% pure by HPLC; peptides were resuspended in DMSO or $\mathrm{ddH}_{2} \mathrm{O}$. Cells were pulsed with $10-100 \mu \mathrm{M}$ of peptide and incubated at $37^{\circ} \mathrm{C}$ for $1-18$ hours in IMDM serum free media before each assay. For T2 cells, 3-35 $\mu \mathrm{M}$ of recombinant $\beta 2 \mathrm{M}$ was also added. As a control, the well-characterized peptide YMLDLQPET (YML) (66) derived from the $\mathrm{E} 7$ protein expressed by HPV-16 was used.

Flow cytometry. Cells were incubated at $4^{\circ} \mathrm{C}$ with $5 \mu \mathrm{g} / \mathrm{ml}$ of each of the antibodies (or isotype matched control) followed by a human Fc-specific (PE-labeled, for human IgG1 Southern Biotech, catalog 204009), mouse Fc-specific (PE-labeled,for BB7.2 or anti-Phage secondary, Southern Biotech, catalog 1010-09), or anti-M13 mAb (mouse IgG1, for Phage staining, GE Healthcare, catalog 27-9420-01) secondary antibody. Samples were acquired using a FACSCalibur flow cytometer (BD Biosciences). Data and overlays were prepared using Flowjo Software Version 6.

Cytotoxicity assays. Cytotoxicity was assayed as previously described (67). Target cells were incubated with $100 \mu \mathrm{Ci}$ of ${ }^{51} \mathrm{Cr}$ and plated at a density of $5 \times 10^{3}$ per well in round bottom 96-well plates. Serial antibody titrations were added along with effector cells. Effector cells were plated at a 20:1 ratio (CD16-transfeceted NK92) or 10:1 (activated human cord blood T cells) to measure ADCC or TDCC, respectively. Cytotoxicity assays were stopped after 4 hours, and chromium release was measured using a gamma counter. Cytotoxicity was calculated with the formula: \% specific lysis $=(($ Experimental Lysis - Spontaneous release)/(Maximum Lysis - Spontaneous release)) - Nonspecific lysis. Maximum lysis was obtained by mixing target cells with SDS, spontaneous release represents the target cells incubated without antibody or effector cells, and nonspecific lysis is obtained by mixing the target and effector cells without antibody. The $\mathrm{EC}_{50}$ and max killing was calculated based on a nonlinear 4-parameter variable slope regression analysis.

Animal xenograft studies. For in vivo therapy studies, DKO mice (derived from colony of Mamoru Ito, Central Institute for Experimental Animals [CIEA], Kawasaki, Japan) $(68,69)$ were used. Tumor cell lines (transduced with luciferase reporter genes) were injected i.v., and growth was monitored by bioluminescence, using the Xenogen In Vivo Imaging System (IVIS) 200 (Caliper LifeSciences). Images were collected 1-2 minutes after injection using the following parameters: a 10- to 60-second exposure time, medium binning, and an $8 \mathrm{f}$ stop. Bioluminescence image analysis was performed using Living Image 2.6 (Caliper LifeSciences). Mice were treated with i.v. administration of human PBMCs and DiBsAb.

Statistics. All data were analyzed using Prism 6 (GraphPad), including nonlinear curve fitting algorithms (log [agonist] vs. response-Variable slope with 4 parameters) to determine $\mathrm{EC}_{50}$ values, and AUC calculations for in vivo bioluminescence measurements. Unpaired 2-tailed Student's $t$ test was used to assess 2 independent groups. Log-rank tests were used to determine significance for Kaplan-Meier survival analysis. $P<0.05$ was considered statistically significant.

Study approval. All animal studies were approved under protocol number 90-09-018 by the IACUC at Memorial Sloan Kettering Cancer Center.

\section{Author contributions}

MA and ALA designed research studies, conducted experiments, acquired and analyzed data, and contributed to writing the manuscript. NKVC designed research studies, analyzed data, and contributed to writing the manuscript. DP, BHS, HL, SY, JX, PW, ANH, and AS conducted experiments and acquired data. RJOR and CL designed research studies and analyzed data.

\section{Acknowledgments}

Technical services provided by the MSKCC Small-Animal Imaging Core Facility, Research Animal Resource Center, Integrated Genomics Core Facility, and Antibody \& Bioresource Core Facility were supported in part by NIH Cancer Center Support grant 2P30CA008748-48. We thank Mamoru Ito for providing the DKO mice for our studies. We thank Vivien Chan for assistance with bioinformatics analysis. We also thank Hong-fen Guo, Yi Feng, and Jian Hu for technical assistance. 
Address correspondence to: Nai-Kong V. Cheung or Mahiuddin Ahmed, Department of Pediatrics, Memorial Sloan Kettering Cancer Center, 1275 York Avenue, New York, New York 10065, USA. Phone: 646.888.2313; Email: cheungn@mskcc.org (NKV Cheung). Phone: 646.888.2320; Email: ahmedm@mskcc.org (M. Ahmed).

1. de-The G, et al. Sero-epidemiology of the Epstein-Barr virus: preliminary analysis of an international study - a review. IARC Sci Publ. 1975;(11 Pt 2):3-16.

2. Hislop AD, Taylor GS, Sauce D, Rickinson AB. Cellular responses to viral infection in humans: lessons from Epstein-Barr virus. Annu Rev Immunol. 2007;25:587-617.

3. Thorley-Lawson DA. EBV Persistence--Introducing the Virus. Curr Top Microbiol Immunol. 2015;390(Pt 1):151-209.

4. Khan G, Hashim MJ. Global burden of deaths from Epstein-Barr virus attributable malignancies 1990-2010. Infect Agents Cancer. 2014;9(1):38.

5. McLaughlin LP, Gottschalk S, Rooney CM, Bollard CM. EBV-Directed T Cell Therapeutics for EBV-Associated Lymphomas. Methods Mol Biol. 2017;1532:255-265.

6. Bollard CM, Rooney CM, Heslop HE. T-cell therapy in the treatment of post-transplant lymphoproliferative disease. Nat Rev Clin Oncol. 2012;9(9):510-519.

7. Rasche L, Kapp M, Einsele H, Mielke S. EBV-induced post transplant lymphoproliferative disorders: a persisting challenge in allogeneic hematopoetic SCT. Bone Marrow Transplant. 2014;49(2):163-167.

8. Luo B, et al. Expression of Epstein-Barr virus genes in EBV-associated gastric carcinomas. World J Gastroenterol. 2005;11(5):629-633

9. Han J, et al. Sequence variations of latent membrane protein $2 \mathrm{~A}$ in Epstein-Barr virus-associated gastric carcinomas from Guangzhou, southern China. PLoS ONE. 2012;7(3):e34276.

10. Dubrovsky L, et al. T cell receptor mimic antibodies for cancer therapy. Oncoimmunology. 2016;5(1):e1049803.

11. Cheever MA, et al. The prioritization of cancer antigens: a national cancer institute pilot project for the acceleration of translational research. Clin Cancer Res. 2009;15(17):5323-5337.

12. Lai J, et al. TCR-like antibodies mediate complement and antibody-dependent cellular cytotoxicity against Epstein-Barr virus-transformed B lymphoblastoid cells expressing different HLA-A*02 microvariants. Sci Rep. 2017;7(1):9923.

13. Lee SP, et al. HLA A2.1-restricted cytotoxic T cells recognizing a range of Epstein-Barr virus isolates through a defined epitope in latent membrane protein LMP2. J Virol. 1993;67(12):7428-7435.

14. Moosmann A, et al. Effective and long-term control of EBV PTLD after transfer of peptide-selected T cells. Blood. 2010;115(14):2960-2970.

15. Sim AC, et al. Defining the expression hierarchy of latent T-cell epitopes in Epstein-Barr virus infection with TCR-like antibodies. Sci Rep. 2013;3:3232.

16. Lai J, et al. Targeting Epstein-Barr virus-transformed B lymphoblastoid cells using antibodies with T-cell receptor-like specificities. Blood. 2016;128(10):1396-1407.

17. Stewart-Jones GB, McMichael AJ, Bell JI, Stuart DI, Jones EY. A structural basis for immunodominant human T cell receptor recognition. Nat Immunol. 2003;4(7):657-663.

18. Garboczi DN, Ghosh P, Utz U, Fan QR, Biddison WE, Wiley DC. Structure of the complex between human T-cell receptor, viral peptide and HLA-A2. Nature. 1996;384(6605):134-141.

19. Ding YH, Smith KJ, Garboczi DN, Utz U, Biddison WE, Wiley DC. Two human T cell receptors bind in a similar diagonal mode to the HLA-A2/Tax peptide complex using different TCR amino acids. Immunity. 1998;8(4):403-411.

20. Chen JL, et al. Structural and kinetic basis for heightened immunogenicity of T cell vaccines. J Exp Med. 2005;201(8):1243-1255.

21. Borbulevych OY, et al. T cell receptor cross-reactivity directed by antigen-dependent tuning of peptide-MHC molecular flexibility. Immunity. 2009;31(6):885-896.

22. Borbulevych OY, Piepenbrink KH, Baker BM. Conformational melding permits a conserved binding geometry in TCR recognition of foreign and self molecular mimics. J Immunol. 2011;186(5):2950-2958.

23. Gras S, et al. Structural bases for the affinity-driven selection of a public TCR against a dominant human cytomegalovirus epitope. J Immunol. 2009;183(1):430-437.

24. Rudolph MG, Wilson IA. The specificity of TCR/pMHC interaction. Curr Opin Immunol. 2002;14(1):52-65.

25. Bargou R, et al. Tumor regression in cancer patients by very low doses of a T cell-engaging antibody. Science. 2008;321(5891):974-977.

26. Zhao Q, et al. Affinity maturation of T-cell receptor-like antibodies for Wilms tumor 1 peptide greatly enhances therapeutic potential. Leukemia. 2015;29(11):2238-2247.

27. Ahmed M, Cheng M, Cheung IY, Cheung NK. Human derived dimerization tag enhances tumor killing potency of a T-cell engaging bispecific antibody. Oncoimmunology. 2015;4(4):e989776.

28. Gorin NC, Piantadosi S, Stull M, Bonte H, Wingard JR, Civin C. Increased risk of lethal graft-versus-host disease-like syndrome after transplantation into NOD/SCID mice of human mobilized peripheral blood stem cells, as compared to bone marrow or cord blood. J Hematother Stem Cell Res. 2002;11(2):277-292.

29. Moutaftsi $M$, et al. A consensus epitope prediction approach identifies the breadth of murine $\mathrm{T}(\mathrm{CD} 8+)$-cell responses to vaccinia virus. Nat Biotechnol. 2006;24(7):817-819.

30. Ataie N, et al. Structure of a TCR-Mimic Antibody with Target Predicts Pharmacogenetics. J Mol Biol. 2016;428(1):194-205.

31. Cameron BJ, et al. Identification of a Titin-derived HLA-A1-presented peptide as a cross-reactive target for engineered MAGE A3-directed T cells. Sci Transl Med. 2013;5(197):197ra103.

32. Dahan R, Reiter Y. T-cell-receptor-like antibodies - generation, function and applications. Expert Rev Mol Med. 2012;14:e6.

33. Denkberg G, Cohen CJ, Lev A, Chames P, Hoogenboom HR, Reiter Y. Direct visualization of distinct T cell epitopes derived from a melanoma tumor-associated antigen by using human recombinant antibodies with MHC- restricted $\mathrm{T}$ cell receptor-like 
specificity. Proc Natl Acad Sci USA. 2002;99(14):9421-9426.

34. Sergeeva A, et al. An anti-PR1/HLA-A2 T-cell receptor-like antibody mediates complement-dependent cytotoxicity against acute myeloid leukemia progenitor cells. Blood. 2011;117(16):4262-4272.

35. Tassev DV, Cheng M, Cheung NK. Retargeting NK92 cells using an HLA-A2-restricted, EBNA3C-specific chimeric antigen receptor. Cancer Gene Ther. 2012;19(2):84-100.

36. Dao T, et al. Targeting the intracellular WT1 oncogene product with a therapeutic human antibody. Sci Transl Med. 2013;5(176):176ra33.

37. Townsend A, Bodmer H. Antigen recognition by class I-restricted T lymphocytes. Annu Rev Immunol. 1989;7:601-624.

38. Rudolph MG, Stanfield RL, Wilson IA. How TCRs bind MHCs, peptides, and coreceptors. Annu Rev Immunol. 2006;24:419-466

39. Parker KC, et al. Sequence motifs important for peptide binding to the human MHC class I molecule, HLA-A2. J Immunol. 1992;149(11):3580-3587.

40. Rudolph MG, Wilson IA. The specificity of TCR/pMHC interaction. Curr Opin Immunol. 2002;14(1):52-65.

41. Degano M, Garcia KC, Apostolopoulos V, Rudolph MG, Teyton L, Wilson IA. A functional hot spot for antigen recognition in a superagonist TCR/MHC complex. Immunity. 2000;12(3):251-261.

42. Reali E, et al. A single specific amino acid residue in peptide antigens is sufficient to activate memory CTL: potential role of cross-reactive peptides in memory T cell maintenance. J Immunol. 1999;162(1):106-113.

43. Andersen PS, Stryhn A, Hansen BE, Fugger L, Engberg J, Buus S. A recombinant antibody with the antigen-specific, major histocompatibility complex-restricted specificity of T cells. Proc Natl Acad Sci USA. 1996;93(5):1820-1824.

44. Willemsen RA, Debets R, Hart E, Hoogenboom HR, Bolhuis RL, Chames P. A phage display selected fab fragment with MHC class I-restricted specificity for MAGE-A1 allows for retargeting of primary human T lymphocytes. Gene Ther. 2001;8(21):1601-1608.

45. Low JL, et al. Binding of TCR multimers and a TCR-like antibody with distinct fine-specificities is dependent on the surface density of HLA complexes. PLoS ONE. 2012;7(12):e51397.

46. Biddison WE, Turner RV, Gagnon SJ, Lev A, Cohen CJ, Reiter Y. Tax and M1 peptide/HLA-A2-specific Fabs and T cell receptors recognize nonidentical structural features on peptide/HLA-A2 complexes. J Immunol. 2003;171(6):3064-3074.

47. Cohen CJ, Hoffmann N, Farago M, Hoogenboom HR, Eisenbach L, Reiter Y. Direct detection and quantitation of a distinct T-cell epitope derived from tumor-specific epithelial cell-associated mucin using human recombinant antibodies endowed with the antigen-specific, major histocompatibility complex-restricted specificity of T cells. Cancer Res. 2002;62(20):5835-5844

48. Cohen CJ, Sarig O, Yamano Y, Tomaru U, Jacobson S, Reiter Y. Direct phenotypic analysis of human MHC class I antigen presentation: visualization, quantitation, and in situ detection of human viral epitopes using peptide-specific, MHC-restricted human recombinant antibodies. J Immunol. 2003;170(8):4349-4361.

49. Epel M, et al. Targeting TARP, a novel breast and prostate tumor-associated antigen, with T cell receptor-like human recombinant antibodies. Eur J Immunol. 2008;38(6):1706-1720.

50. Zhang G, et al. Anti-melanoma activity of T cells redirected with a TCR-like chimeric antigen receptor. Sci Rep. 2014;4:3571

51. Klechevsky E, et al. Antitumor activity of immunotoxins with T-cell receptor-like specificity against human melanoma xenografts. Cancer Res. 2008;68(15):6360-6367.

52. Oren R, et al. Functional comparison of engineered T cells carrying a native TCR versus TCR-like antibody-based chimeric antigen receptors indicates affinity/avidity thresholds. J Immunol. 2014;193(11):5733-5743.

53. Stewart-Jones G, et al. Rational development of high-affinity T-cell receptor-like antibodies. Proc Natl Acad Sci USA. 2009;106(14):5784-5788.

54. Verma B, et al. TCR mimic monoclonal antibodies induce apoptosis of tumor cells via immune effector-independent mechanisms. J Immunol. 2011;186(5):3265-3276.

55. Weidanz JA, et al. Levels of specific peptide-HLA class I complex predicts tumor cell susceptibility to CTL killing. J Immunol. 2006;177(8):5088-5097.

56. Zhang G, et al. Anti-melanoma activity of T cells redirected with a TCR-like chimeric antigen receptor. Sci Rep. 2014;4:3571.

57. Ziegler A, Coulie PG, Uchańska-Ziegler B. Monoclonal and recombinant antibodies with T cell receptor-like reactivity. Recent Results Cancer Res. 2007;176:229-241.

58. van den Berg JH, et al. Case Report of a Fatal Serious Adverse Event Upon Administration of T Cells Transduced With a MART-1-specific T-cell Receptor. Mol Ther. 2015;23(9):1541-1550.

59. Burrows SR, Rossjohn J, McCluskey J. Have we cut ourselves too short in mapping CTL epitopes? Trends Immunol. 2006;27(1):11-16

60. Vitiello A, Marchesini D, Furze J, Sherman LA, Chesnut RW. Analysis of the HLA-restricted influenza-specific cytotoxic T lymphocyte response in transgenic mice carrying a chimeric human-mouse class I major histocompatibility complex. J Exp Med. 1991;173(4):1007-1015.

61. Altman JD, Davis MM. MHC-peptide tetramers to visualize antigen-specific T cells. Curr Protoc Immunol. 2003; Chapter 17:Unit 17.3.

62. Tomimatsu K, et al. Production of human monoclonal antibodies against Fc(epsilon)RI(alpha) by a method combining in vitro immunization with phage display. Biosci Biotechnol Biochem. 2009;73(7):1465-1469.

63. Strong MJ, Baddoo M, Nanbo A, Xu M, Puetter A, Lin Z. Comprehensive high-throughput RNA sequencing analysis reveals contamination of multiple nasopharyngeal carcinoma cell lines with HeLa cell genomes. J Virol. 2014;88(18):10696-10704.

64. Menezes J, Leibold W, Klein G, Clements G. Establishment and characterization of an Epstein-Barr virus (EBC)-negative lymphoblastoid B cell line (BJA-B) from an exceptional, EBV-genome-negative African Burkitt's lymphoma. Biomedicine. 1975;22(4):276-284.

65. Hasan AN, Selvakumar A, Doubrovina E, Riviere I, Sadelain MW, O'Reilly RJ. Artificial antigen presenting cells that express prevalent HLA alleles: A step towards the broad application of antigen-specific adoptive cell therapies. Discov Med. 2009;8(43):210-218

66. Tsang KY, et al. Identification and characterization of enhancer agonist human cytotoxic T-cell epitopes of the human papillomavirus type 16 (HPV16) E6/E7. Vaccine. 2017;35(19):2605-2611.

67. Xu H, Cheng M, Guo H, Chen Y, Huse M, Cheung NK. Retargeting T cells to GD2 pentasaccharide on human tumors using Bispecific humanized antibody. Cancer Immunol Res. 2015;3(3):266-277. 
68. Koo GC, Hasan A, O'Reilly RJ. Use of humanized severe combined immunodeficient mice for human vaccine development. Expert Rev Vaccines. 2009;8(1):113-120.

69. Andrade D, et al. Engraftment of peripheral blood mononuclear cells from systemic lupus erythematosus and antiphospholipid syndrome patient donors into BALB-RAG-2-/- IL-2R $\gamma$-/- mice: a promising model for studying human disease. Arthritis Rheum. 2011;63(9):2764-2773. 